\title{
Role of Temporal Discounting in a Conditional Cash Transfer (CCT) Intervention to Improve Engagement in the Prevention of Mother-to-Child Transmission (PMTCT) Cascade
}

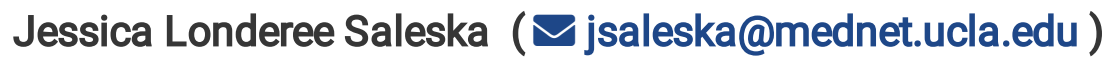

University of California Los Angeles https://orcid.org/0000-0002-2733-724X

Abigail Norris Turner

The Ohio State University

Maria F. Gallo

The Ohio State University

Abigail Shoben

The Ohio State University

Bienvenu Kawende

The University of Kinshasa

Noro Lantoniaina Rosa Ravelomanana

The University of Kinshasa

Harsha Thirumurthy

University of Pennsylvania Perelman School of Medicine

Marcel Yotebieng

Albert Einstein College of Medicine

Research

Keywords: Temporal discounting, HIV, Prevention of Mother-to-Child Transmission of HIV (PMTCT), PMTCT Cascade, Intertemporal Preferences

Posted Date: June 5th, 2020

DOI: https://doi.org/10.21203/rs.3.rs-32878/v1

License: (c) (i) This work is licensed under a Creative Commons Attribution 4.0 International License. Read Full License 


\section{Abstract}

Background: Temporal discounting (TD), the tendency of individuals to discount future costs and benefits relative to the present, is often associated with greater engagement in risky behaviors. Incentives such as conditional cash transfers (CCTs) have the potential to counter the effects of TD on health behaviors. We hypothesized that CCTs work by mitigating the effects of high TD, measured using a delay-discounting task.

Methods: We conducted an interaction analysis using data from a randomized trial of a CCT intervention among 434 HIV-positive pregnant women in the Democratic Republic of Congo. The analysis focused on two outcomes: 1) retention in HIV care, and 2) uptake of prevention of mother-to-child transmission (PMTCT) services.

Results: The effect of TD on retention was small, and we did not observe evidence of interaction between TD and CCT on retention. However, our findings suggest that CCT may mitigate the negative effect of high TD on uptake of PMTCT services (interaction contrast (IC): $0.18,95 \% \mathrm{Cl}:-0.09,0.44$ ).

Conclusions: Our findings support the continued use of small, frequent incentives, to motivate improved uptake of PMTCT services, especially among women exhibiting high TD.

\section{CONTRIBUTIONS TO THE LITERATURE}

Conditional cash transfer (CCT) interventions demonstrate varying degrees of effectiveness in improving engagement in HIV care. Understanding how these interventions work allow for better adaptation in different contexts, though few studies have explored the underlying mechanisms of CCT.

We assessed whether CCT works by mitigating the effect of temporal discounting on disengagement from care, a major posited mechanism of the intervention, among HIV infected pregnant and breastfeeding women in the Democratic Republic of Congo.

Our findings contribute to gaps in literature surrounding the role of temporal discounting, and its mitigation by CCT, on engagement in HIV care.

\section{Background}

The rapid scale-up of interventions to prevent mother-to-child transmission of HIV over the past decade has led to drastic reductions in the number of new HIV-1 infections among children, which fell by an estimated 35\% between 2010 and 2017 [1]. This drop is largely attributed to expansions in access to antiretroviral therapy (ART) among pregnant and breastfeeding women. If taken throughout pregnancy and breastfeeding, ART can reduce the risk of vertical HIV transmission to approximately 1\% [1], compared to $20-25 \%$ in the absence of any intervention [2]. Nonetheless, despite broad access to ART, approximately 160,000 children were infected with HIV worldwide in 2018 [3]. 
The continued occurrence of new HIV infections among children has been attributed to inadequate adherence to ART and poor engagement with the series of steps in care necessary to maximize maternal and infant health outcomes, known as the prevention of mother-to-child transmission (PMTCT) cascade [4]- [8]. The PMTCT cascade includes attendance at regular clinic visits (i.e., retention in care, at a minimum for ART refills), delivery in a health facility, and testing of the HIV-exposed infant at specific post-partum time points (six weeks, nine months, and at breastfeeding cessation).

In many countries with high HIV prevalence, only a limited number of women and their infants complete the full PMTCT cascade [8]-[10]. Failure to complete the cascade can hinder ART adherence, which can in turn lead to suboptimal viral suppression [11], increased risk of vertical transmission [5], and the development of drug resistance [12], [13]. Further, failure to access adequate antenatal care (including HIV services, as appropriate), or deliver within a health facility, substantially increases the risk of maternal mortality among HIV-infected women [14].

Insight from many disciplines, both within and outside of public health, can inform novel approaches to identify and mitigate the factors contributing to disengagement from the PMTCT cascade. The field of economics, for example, has gained considerable recognition for its ability to help understand choices surrounding HIV prevention and treatment [15], [16]. Specifically, the study of decisions involving tradeoffs between immediate vs. future costs and benefits - referred to as intertemporal preferences provides insight into decision-making processes that lead to certain risky behaviors, including poor retention to care [15]-[18]. Of key consideration in the study of intertemporal preferences is temporal discounting (TD), or the tendency to discount the utility, or value, of future costs and benefits relative to those in the present. High levels of TD could influence actions surrounding HIV care by causing an individual to value the immediate benefits of disengaging from care (e.g., avoiding stigma and medication side effects or opportunity cost to attend regular clinic visits) over the substantial future benefits of completing the full PMTCT cascade (e.g., PMTCT and disease management). Similarly, individuals may also discount future costs associated with not seeking care today (such as poorer health in the future).

Behavioral interventions such as CCTs could mitigate the negative impact of high TD on a variety of health behaviors. СCT interventions involve cash payments to individuals or households on the condition that recipients take certain actions or engage in healthy behaviors [19]. Through the provision of these frequent, small incentives, CCT can enhance the immediate rewards, and reduce the immediate costs, associated with adherence and retention [20]. In doing so, CCT could lead to improved engagement in HIV care, especially among those who exhibit high temporal discounting [15].

In randomized controlled trials (RCTs) evaluating CCT and other interventions involving conditional economic incentives to improve engagement in HIV care services, however, results demonstrate varying degrees of efficacy [21]-[28]. Exploring why CCT does or does not work in practice warrants a deeper understanding of how such intervention may affect the mechanisms underlying low engagement HIV care. Nonetheless, few studies to date explore how CCT works to promote retention and engagement in 
HIV care [29]. None have examined whether the effects of CCTs are moderated by individuals' intertemporal preferences, even as inordinately high TD from the present is often cited as one important mechanism of action of CCT in this context [29]. Assessing the modifying role of CCT on the association between intertemporal preferences and risky health behavior could provide insight into how CCT works to improve health outcomes. The objective of the present study is then to examine the impact of CCT on the effect of high TD on engagement at key points in the PMTCT cascade (i.e., through retention and uptake of available PMTCT services) throughout pregnancy and breastfeeding.

\section{Methods}

We performed a secondary analysis of data from an RCT conducted in Kinshasa, the Democratic Republic of Congo. The parent study's objective was to assess the effect of a CCT intervention on retention throughout the PMTCT care continuum and uptake of available services among newlydiagnosed HIV-infected pregnant women. Between April 2013 and August 2014, 434 pregnant women were enrolled from 89 maternal and child health clinics throughout Kinshasa. Participants were enrolled at $£ 32$ weeks of gestation and randomized to receive either standard of care or modest and increasing cash incentives (starting at $\$ 5$ and increasing by $\$ 1$ each visit), conditional on attending scheduled clinic visits, providing a blood sample for a CD4+ T-cell count, accepting referral for ART if referred, delivering in a health facility, and providing a blood sample for infant HIV diagnosis at 6 weeks postpartum. A comprehensive description of the parent study methods and results, which showed that the CCT intervention increased retention in HIV care, has been published previously [30]. All participants included in the parent study were eligible for this analysis. Reporting of our study was guided by the Strengthening the Reporting of Observational Studies in Epidemiology (STROBE) guidelines.

\section{Outcomes:}

We assessed two primary outcomes: 1) retention to HIV care, and 2) complete uptake of all available PMTCT services. Women were classified as retained if they were in HIV care (i.e., present at scheduled clinic visit) at 6 weeks postpartum, whereas women who were not present in care at 6 weeks postpartum were classified as not retained, regardless of the reasons for not being in care (e.g., poor pregnancy outcome). We determined complete uptake of available PMTCT services based on participants' completion of all the following conditions: attendance at all scheduled clinic visits (within five days of the scheduled date) from randomization through six weeks postpartum (including giving birth in a study clinic) and acceptance of all recommended services including providing blood samples for CD4+ T-cell count and dried blood spot sample for DNA PCR testing at 6 weeks.

\section{Temporal discounting:}

To assess TD, participants were told to suppose they had won a prize. Then, they were asked to choose among a series of two hypothetical monetary options for payment, the first being a smaller reward offered immediately, and the second being a larger reward which would be given to the participant after one year. This series, known as a delay-discounting task, operated on a gradient, with a steadily greater 
reward offered in one year ( $\$ 55$ today vs. $\$ 60$ in one year, $\$ 55$ today vs. $\$ 80$ in one year, $\$ 55$ today vs. $\$ 100$ in one year). The task concluded with the following question for the interviewer: "Did the respondent understand the questions above?" Respondents were excluded from the analysis if the interviewer indicated that they had not understood the task.

The extent of TD was determined by an individual's discount rate, or the proportional change in the perceived utility of a given reward through time. Responses to the delay-discounting task in this study indicate a participant's discount rate from the present to one year: the choice of the present smaller reward over the increasing larger reward in one year reflects a higher and higher discount rate. For example, an individual who chooses $\$ 55$ today over $\$ 60$ in one year, but not over $\$ 80$ in one year, would exhibit a lower one-year discount rate than an individual who chooses $\$ 55$ today over $\$ 80$ in one year. Given the distributions of responses to the delay-discounting task, we classified women into two discount groups: the high discount rate group included women who consistently chose the immediate reward of $\$ 55$ irrespective of the value of future reward. The low discount rate group included women who chose at least one of the larger rewards that were offered in one year.

\section{Covariates:}

At enrollment in the study, participants were also interviewed using a structured questionnaire to collect information on food insecurity, depression symptoms and various demographic factors. We evaluated depression symptoms using the Patient Health Questionnaire (PHQ-9) [31], and assessed food insecurity using questions adapted from the U. S. Department of Agriculture (USDA)'s food security core-module questionnaire [32]. Depression symptoms were dichotomized into low to moderate depressive symptoms (PHQ-9 score of $<15$ ) and severe depressive symptoms (PHQ-9 score of $\geq 15$ ). Similarly, food insecurity was dichotomized into low to moderate food insecurity vs. severe food insecurity. A relative financial status index variable was calculated via a factor analysis of numerous measures related to financial status, including education, household crowding (i.e., proportion of people living in the house per household room), number of beds in a household, cooking fuel type, household water source and ownership for several assets. The factor score was then categorized into 3 categories: upper (2 richest quintiles), middle ( $3^{\text {rd }}$ quintile) and lower (2 poorest quintiles).

In addition to self-reported demographic and behavioral measures, we also assessed disease severity at enrollment by measuring CD4+ T-cell count (i.e., CD4+ T-cells per milliliter of blood). CD4+ T-cell count was dichotomized into $£ 350$ cells $/ \mathrm{mL}$ and $>350$ cells $/ \mathrm{mL}$ categories. Self-reported alcohol use (no reported use vs. reported use), age at enrollment (in years) and CCT intervention arm were also included in the analysis.

\section{Analysis:}

Quantification of the effect of high TD on retention and complete uptake of services 
Before assessing whether CCT mitigates the effect of high TD on retention and uptake of PMTCT services, it was necessary to ascertain whether high TD has a significant effect on these outcomes. We examined the associations between high TD with retention and complete uptake of services by comparing the proportion of participants retained in care or with complete uptake of services by six weeks postpartum across the two levels of TD (high vs. low) using Pearson's $\chi^{2}$ tests. Next, we used unadjusted and adjusted log-binomial risk models to calculate RRs assessing the effect of high TD on retention and complete uptake of PMTCT services at 6 weeks postpartum. For the adjusted analysis, we included several potential confounders based on a priori hypotheses and previous research on factors affecting both temporal discounting and retention to care, including disease severity (i.e., CD4+ T-cell count), food insecurity, alcohol use in pregnancy, and age [9], [33], [34]. We then used a backward elimination, change-in-estimate strategy to eliminate hypothesized confounding variables that did not substantially affect the effect estimate (the change-in estimate criterion was $10 \%$ ) to generate the most parsimonious model that captured the effect of TD on retention and complete uptake of services. Further, we included CCT arm and wealth, which were strong predictors of retention and uptake of PMTCT services in previous analyses of this data [30].

\section{Interaction analysis}

To assess potential interaction of the effect of high TD by the CCT intervention, linear risk models were used to estimate the risk difference (RD) measuring the individual and joint effects of the CCT intervention and the high TD on each outcome. The likelihood ratio test was used to assess RD modification, and excess risk due to interaction (interaction contrast [IC] and $95 \% \mathrm{Cl}$ ) was used to assess for potential effect modification. IC is the difference between the observed RD comparing the doublyexposed category (high TD and receipt of CCT) with the doubly-unexposed category (low TD and no CCT or standard of care group), and the expected RD comparing the same two groups (doubly-exposed and doubly-unexposed) assuming additivity (without the interaction term). This difference should be equal to zero in the absence of RD modification. We performed all statistical analyses using SAS version 9.4 (SAS Institute Inc., Cary, NC).

\section{Results}

From the full sample of 434 newly-diagnosed HIV-infected women in the parent study, two women were excluded because the interviewer determined that they had not understood the delay-discounting task, leaving 432 women ( $99.5 \%$ of enrolled participants) in the final analytic sample. Of these remaining women, 332 (76.8\%) were retained in care and $263(60.9 \%)$ exhibited complete uptake of services at 6 weeks postpartum. The majority ( $n=374,86.6 \%)$ of women exhibited high TD.

Temporal discounting did not vary by baseline age, alcohol use in pregnancy, wealth or CD4+ T-cell count (see Table 1). Fewer women with a high discount rate were food insecure, compared to the proportion of women with a low discount rate who were food insecure ( $47.9 \%$ vs. $67.2 \% ; p=0.01)$. Similarly, a lower proportion of women with a high discount rate exhibited symptoms of severe depression (PHQ-9 score of 
$\geq 15)$ compared to the proportion of women with a low discount rate who had symptoms of severe depression ( $9.6 \%$ vs $25.9 \%$; $p<0.001$ ) (see Table 1 ). 
Table 1

Characteristics of participants, by strata of temporal discounting ( $\mathrm{N}=432)$

\section{Temporal Discounting}

\begin{tabular}{llll}
$\begin{array}{l}\text { Total } \\
(\mathbf{N}=432)\end{array}$ & $\begin{array}{l}\text { Low } \\
(\mathbf{n = 5 8 )}\end{array}$ & $\begin{array}{l}\text { High } \\
(\mathbf{n = 3 7 4})\end{array}$ & $\begin{array}{l}\text { - } \\
\text { value }^{\mathrm{b}}\end{array}$ \\
\hline $29(25-34)$ & $\begin{array}{l}29.5(27- \\
34)\end{array}$ & $29(25-34)$ & 0.34 \\
$N \quad \%$ & $N \quad \%$ & $N \quad \%$ & \\
\hline
\end{tabular}

Age: median (IQR)

Retention at 6 weeks postpartum ${ }^{a}$

Retained

Not retained

(Notretaned

Uptake of PMTCT services ${ }^{a}$

\section{Complete Uptake}

Incomplete Uptake

$158 \quad 72.8$

$24 \quad 77.4$

$59 \quad 27.2$

$7 \quad 22.6$

\begin{tabular}{ll}
117 & 53.9 \\
\hline 100 & 46.1
\end{tabular}

\begin{tabular}{ll}
23 & 74.2 \\
\hline 8 & 25.8 \\
\hline
\end{tabular}

CD4+ T-cell Count

$£ 350$ cells $/ \mathrm{mL}$
$>350$ cells $/ \mathrm{mL}$
Missing

Wealth Index

Upper

Middle

\begin{tabular}{lllllll|} 
& & & & & & 0.66 \\
\hline 167 & 38.7 & 26 & 44.8 & 141 & 37.7 & \\
\hline 208 & 48.1 & 29 & 50.0 & 179 & 47.9 & \\
\hline 57 & 13.2 & 3 & 5.2 & 54 & 14.4 &
\end{tabular}

\begin{tabular}{lll}
92 & 49.5 & 0.01 \\
\hline 94 & 50.5 &
\end{tabular}

$52 \quad 28.0$

0.53




\begin{tabular}{|c|c|c|c|c|c|c|c|}
\hline & & & Ter & oral D & Inting & & \\
\hline & $\begin{array}{l}\text { Tota } \\
(\mathrm{N}=4\end{array}$ & & $\begin{array}{l}\text { Lou } \\
(\mathrm{n}=\end{array}$ & & $\begin{array}{c}\text { High } \\
(n=3\end{array}$ & & $\begin{array}{l}\text { p- } \\
\text { value }^{b}\end{array}$ \\
\hline Lower & 173 & 40.0 & 28 & 48.3 & 145 & 38.8 & \\
\hline Food Insecurity (FI) & & & & & & & 0.01 \\
\hline Low FI & 205 & 47.4 & 17 & 39.3 & 188 & 50.2 & \\
\hline Moderate to high FI & 218 & 50.5 & 39 & 67.2 & 179 & 47.9 & \\
\hline Missing & 9 & 2.1 & 2 & 3.5 & 7 & 1.9 & \\
\hline Study Group & & & & & & & 0.60 \\
\hline Intervention & 215 & 49.8 & 27 & 46.6 & 188 & 50.3 & \\
\hline Control & 217 & 50.2 & 31 & 53.4 & 186 & 49.7 & \\
\hline Depression Symptoms & & & & & & & $<0.001$ \\
\hline PHQ-9 score $<15$ & 381 & 88.2 & 43 & 74.1 & 338 & 90.4 & \\
\hline PHQ-9 score $\geq 15$ & 51 & 11.8 & 15 & 25.9 & 36 & 9.6 & \\
\hline Alcohol use in pregnancy & & & & & & & 0.14 \\
\hline Yes & 108 & 25.0 & 19 & 32.8 & 89 & 23.8 & \\
\hline No & 324 & 75.0 & 39 & 67.2 & 285 & 76.2 & \\
\hline
\end{tabular}

IQR: Interquartile Range; CCT: Conditional Cash Transfer; PHQ-9: Patient Health Questionnaire.

${ }^{a}$ Analysis limited to individuals who did not receive the conditional cash transfer intervention $(n=217)$ in order to avoid possible mixing of effects.

${ }^{b}$ For continuous variables, p-values were calculated using the Wilcoxon Rank Sum test. For categorical variables, $p$-values were calculated using Pearson's $\chi^{2}$ test. All tests used complete case analysis (i.e., omitting missing observations) 


\section{The effect of TD on retention and uptake of PMTCT services}

The proportion of participants retained in care at six-weeks postpartum was lower among those with high compared with low TD (72.0 \% vs $77.4 \%$; Table 1), though we did not observe evidence of an association between high TD and retention in unadjusted analysis (RR: 0.64; 95\% Cl:0.32, 1.25; see Table 2). For the multivariable analysis of retention, alcohol use in pregnancy, wealth and severe depression were identified as potential confounders using the change-in-estimate strategy. Adjustment for CCT arm, alcohol use in pregnancy, wealth and severe depression did not alter the association between high TD and retention (RR: $0.64 ; 95 \% \mathrm{Cl}: 0.31-1.32$; see Table2).

Table 2

nadjusted and adjusted risk ratios (RR) for the effect of high temporal discounting (TD) on retention and complete uptake of PMTCT services

$\begin{array}{lll}\text { TD } & \text { RR }(95 \% \mathrm{Cl}) & \begin{array}{l}\text { Adjusted RR }(95 \% \\ \text { Cl) }\end{array}\end{array}$

\section{Outcomes}

Retention

$\begin{array}{lllll}\text { High } & 0.64 & \begin{array}{l}(0.32- \\ 1.28)\end{array} & 0.64^{\mathrm{a}} & (0.31-1.32) \\ \text { Low } & 1 & \text { (ref) } & 1 & \text { (ref) }\end{array}$

Complete uptake of PMTCT services

$\begin{array}{lllll}\text { High } & 0.63 & \begin{array}{l}(0.41- \\ 0.95)\end{array} & 0.61^{\mathrm{b}} & (0.40-0.93) \\ \text { Low } & 0 & \text { (ref) } & 1 & \text { (ref) }\end{array}$

\section{Cl: Confidence Interval}

${ }^{a}$ Adjusted for CCT arm, financial status index, depression symptoms and alcohol use in pregnancy.

${ }^{\mathrm{b}}$ Adjusted for CCT arm and financial status index. 
The proportion of participants who exhibited complete uptake of PMTCT services care was considerably lower among those with high compared with low TD (49.5\% vs $74.2 \%$; Table 1). In unadjusted analysis, women with high TD were less likely to exhibit complete uptake of available PMTCT services compared to women with low TD (RR: 0.63; 95\% Cl: 0.41, 0.95; see Table 2). No confounders were identified using the change-in-estimate strategy for uptake of available services, and adjustment for CCT arm and wealth did not meaningfully alter the association between high TD and complete uptake of available PMTCT services (RR: 0.61; 95\% Cl: 0.40, 0.93; see Table 2).

\section{Modification of the effect of TD on retention and uptake of PMTCT services by CCT}

Participants who had high TD and did not receive CCT had exhibited slightly lower retention risk relative to their counterparts with low TD (RD (95\% Cl): $-0.05(-0.24,0.14)$ Table 3$)$. We found no or little evidence that receiving CCT modified the effect of high TD on retention, though the confidence intervals were wide (IC (95\% Cl): -0.08 (-0.30, 0.14); Table 3). Regarding uptake of PMTCT services, compared to participants with low TD who did not receive CCT (i.e., doubly unexposed), those with high TD were less likely to exhibit complete uptake of PMTCT services (RD (95\% Cl): -0.24 (-0.44, -0.04)), and receiving CCT substantially attenuated this negative effect of high $\operatorname{TD}(0.17(-0.15,0.48)$ Table 3$)$. 
Table 3

Risk differences (RD) and interaction contrasts (IC) for retention and uptake of PMTCT services at 6 weeks postpartum by strata of temporal discounting levels among 432 newly identified HIV-infected women in Kinshasa, Democratic Republic of Congo

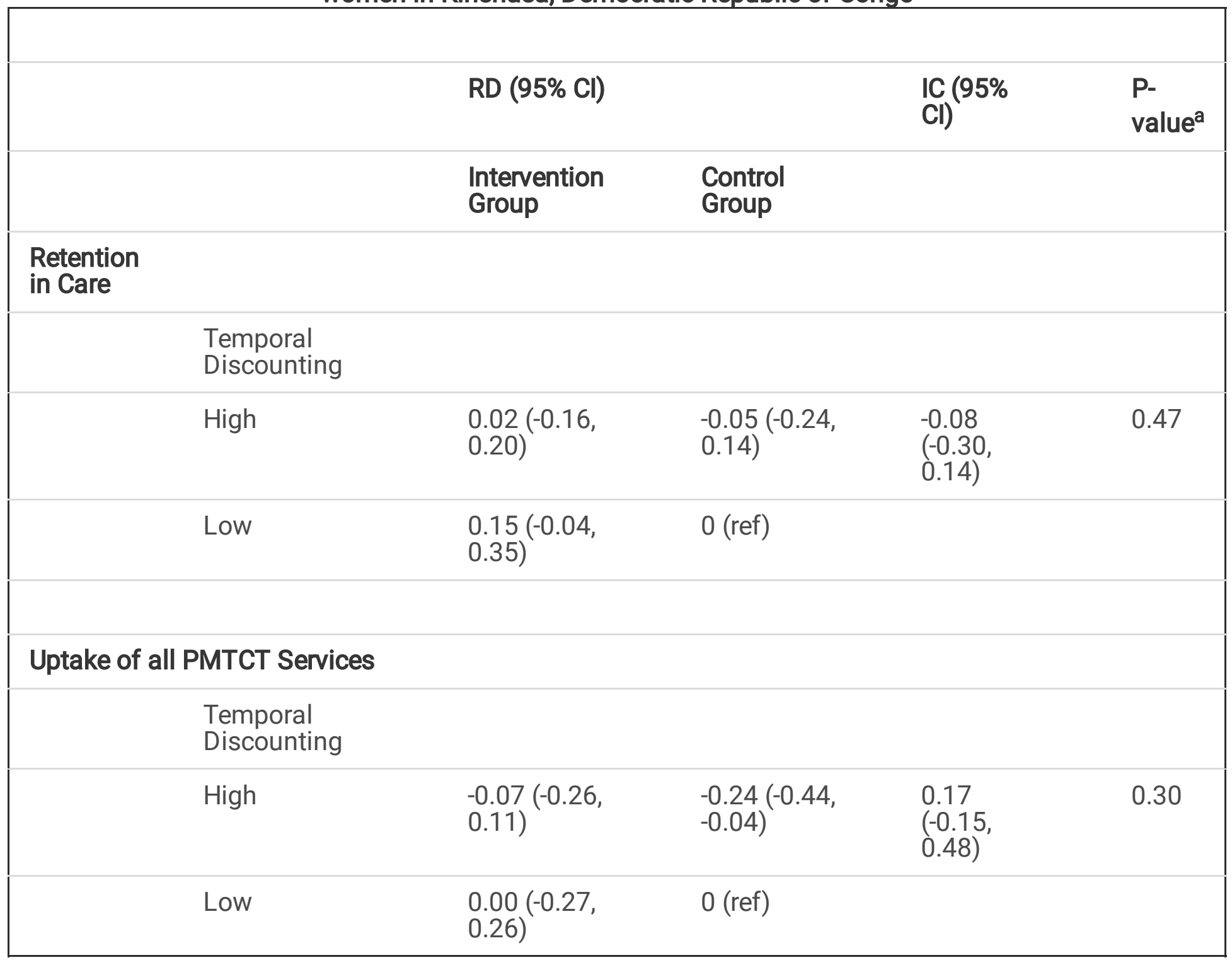

Cl: Confidence Interval; CCT: Conditional Cash Transfer

a $\mathrm{p}$-value reflects the significance of the IC.

\section{Discussion}

Among recently diagnosed HIV-infected women in the Democratic Republic of Congo, we found that complete uptake of recommended PMTCT services was considerably lower among women with high TD, even after adjusting for covariates. Further, in alignment with our hypothesis concerning the mechanisms by which CCTs affect health behaviors, we observed evidence suggesting that the negative effects of high TD on complete uptake of services were mitigated by CCT. Though our results suggest a similar antagonist of CCT on the negative effect of TD on retention in care, our study did not, however, have enough power to access modification of such a small effect. 
Measures of both morbidity and mortality are believed to contribute to high levels of TD, as they reduce utility and opportunity for consumption in the future, respectively [33]-[35]. Thus, it was surprising that our analyses revealed no significant association between discounting and several factors that could affect one's health and survivability, including wealth and food insecurity. Indeed, the negative association between food insecurity and high discount rate was contrary to what we expected given previous research [33]. One possible explanation for these findings is that, while poverty could lead to high discount rates, high discount rates could positively affect wealth accumulation (i.e., inverse causality). Though high levels temporal discounting has often been linked to behaviors that perpetuate the cycle of poverty and ill health among low-income populations [34], discounted utility can - to some extent - be fiscally prudent. The grounds for this prudency are: 1) positive interest rates (i.e., \$55 received now would accrue interest and could be worth more than $\$ 60$ in one year), and 2) future uncertainty (i.e., given the possibility of events that may threaten future utility, $\$ 55$ today carries definite value, whereas the value of $\$ 60$ in one year is uncertain) [36]. Thus, placing more value on immediate rewards relative to those received in one year could - to some degree - be a rational adaptive response, particularly in situations of extreme uncertainty.

Another possible explanation for the negative association between discounting and food insecurity, and the lack of association between discounting and wealth, may lie in the unique life-situation faced by our study population at enrollment: pregnancy. It could be possible that pregnant women experiencing scarcity may prefer to receive money at a later date, after their delivery, in order to ensure they have adequate resources to support their baby. Though poverty and food insecurity could lead to high discount rates, this effect could be tempered or counteracted by fear for the well-being of their unborn child. It is difficult to assess if this might be occurring, however, without data on parental concern or a comparison group of non-pregnant women.

With regards to the impact of TD on our key outcomes, the stronger association between TD and uptake of available services, relative to retention to care, could suggest that TD is more closely linked to decisionmaking upon attendance at clinic appointments, including the acceptance of all proposed services (e.g., provision of blood samples). As with retention, no study to date has assessed the impact of temporal discounting on uptake of PMTCT services, so we are unable to compare our results to other findings. Several studies, however, have assessed the effect of discounting on adherence to ART (i.e., taking the ART medication as prescribed): in a study of HIV-infected adults in Uganda, investigators Linnemayr and Stecher found that individuals with a high discount rate exhibited significantly lower medication adherence than those with a low discount rate [39]. Similarly, in a study of HIV-positive adults on ART in Kenya, Thirumurthy et al. reported lower medication adherence among those with a high discount rate, though this association was not statistically significant, and that high discount rates were associated with significantly higher risk of mortality [40]. Though not directly comparable to our results surrounding uptake of PMTCT services, these previous findings may also suggest that TD may influence individuals' decisions surrounding HIV care, beyond attendance at clinic visits. 
Acceptance of the necessary testing and treatment, in addition to retaining in care, represent crucial steps within the PMTCT cascade [41]. Uptake of these services may be especially challenging in the Democratic Republic of Congo, as many women may be afraid to publicly or privately acknowledge their diagnosis; HIV is highly stigmatized and is often perceived as a moral failing, thus accepting HIV-specific services (and acknowledging one's diagnosis in doing so) can have damaging social and personal implications [42], [43]. Retention, by contrast, is measured by attendance at routine clinic visits for prenatal and infant care, and thus does not implicate an acknowledgement of - and reaction to - one's HIV status. Strong sources of external motivation may then be necessary for many women to overcome their fears surrounding the acknowledgement of their HIV status via uptake of HIV-specific services, beyond retention in care.

One such source of motivation may be the coverage of transport costs upon arrival for routine clinic visits. In a previous study using the same data, Yotebieng et al. found evidence suggesting that CCT improves retention by mitigating the cost of clinic attendance [44]. Among women wishing to attend clinic visits as part of routine prenatal or infant care, but who are unable to do so because of travel difficulties, CCT could provide enough money to cover the cost of transport. Women exhibiting high levels of TD from the present may experience a strong impetus to accept HIV-related services, as they are offered immediate monetary incentive for these behaviors and may overweight the value of this incentive. The immediate costs of clinic attendance, too, may be over-weighted among these women, and so they may be more likely to accept HIV-services to recoup their losses. These sources of motivation, rooted in the provision of CCT, could potentially overcome the objections to acceptance HIV-related services among those who are afraid to acknowledge their HIV status.

Our findings signify qualitative trends that could provide evidence for our hypothesis; however, they do not reflect definitive findings regarding the mechanisms of CCT. We interpreted our results as indicating potential antagonism between high TD and CCT on uptake of services, despite the limited statistical power of this study. Though moderate in size, the parent study was designed to detect the main effects of CCT, not interaction between TD and CCT, and only a small proportion of the study population exhibited low TD.

Further, we were unable to directly assess whether participants displayed time-inconsistent (or present biased) preferences, which could be an additional barrier to behaviors involving immediate costs and delayed benefit [45]. Though high discounting from the present could be indicative of present bias, it may also reflect high levels of time-consistent discounting (i.e., general rates of discounting, rather than an inordinate preference for the present) [46], [47]. It should also be noted that our findings reflect the context, population and parameters of this specific CCT intervention, and may not be generalizeable to other interventions involving economic incentives. There is some disagreement as to the appropriate method to measure TD, especially within low-income countries. Most measures of discounting were developed for a Western setting, and few studies have evaluated the use of existing measures across different cultural and economic contexts. Some argue that effort tasks (i.e., where individuals are asked to choose between earlier or later activities) should be preferred over monetary tasks (i.e., where 
individuals are asked to choose between earlier or later amounts of money), especially in low-income settings [48], [49]. The rationale for this argument is that the monetary discount rates in low-income contexts may reflect transient economic needs and not general intertemporal preferences. Recent studies have provided compelling evidence that, for those in poverty whose income is variable and subject to shocks, measures of discounting may capture volatile economic circumstances and not entrenched temporal preferences [50], [51]. As we did not measure discounting levels before and after shifts in economic circumstances, we do not know whether our measures of discounting reflect intrinsic preferences or situational constraints. Nonetheless, our study is among the first to explore any potential mechanisms by which CCT are hypothesized to improve HIV treatment and prevention [52] and high monetary discounting, regardless of cause or permanence, could arguably have similar effects on behavior in the immediate future when monetary rewards are offered, as is the case in our study.

\section{Conclusions}

By identifying the underlying mechanisms of behavior change, and assessing whether a given intervention can influence these mechanisms, public health practitioners can develop more effective strategies to initiate and maintain healthy behaviors (i.e., a mechanisms-focused approach to behavior change research) [53]. Understanding how interventions such as CCT work could identify populations that would most benefit from such an intervention, and permit better adaptation of the intervention into different settings and contexts. From a broader perspective, improving retention and uptake of services among pregnant, HIV-positive women is critical to ensure that PMTCT efforts achieve maximum effectiveness [4]. Our findings support the continued use of small, frequent incentives, to motivate improved uptake of PMTCT services, especially among women exhibiting high TD.

\section{List Of Abbreviations}

ART: Antiretroviral Therapy; PMTCT: Prevention of Mother to Child Transmission of HIV; TD: Temporal Discounting; CCT: Conditional Cash Transfer; RCT: Randomized Controlled Trial; RR: Risk Ratio; RD: Risk Difference; Cl: Confidence Interval; IC: Interaction Contrast

\section{Declarations}

\section{Ethics approval and consent to participate}

Approval to conduct the study was gained from the Institutional Review Board of the Ohio State University and the Ethical Committee of the Kinshasa School of Public Health. All participants provided informed consent before participating in the study.

\section{Consent for publication}

Not applicable 
Availability of data and materials

The datasets generated and analyzed in the current study are not publicly available in order to maintain confidentiality of study participants but are available from the corresponding author on reasonable request.

\section{Competing interests}

The authors declare that they have no competing interests.

\section{Funding}

This parent trial is registered at ClinicalTrials.gov, number NCT01838005. The trial was supported by a grant from the President's Emergency Plan for AIDS Relief (PEPFAR) and the National Institute of Health and Child Development: NIHCD 1R01 HD075171. The funding body was not involved in the design of the study and collection, analysis, and interpretation of data and in writing of the present manuscript.

\section{Author contributions}

MY, BK and NLRR collected and cleaned the data. JLS, MY, AS and ANT developed the methodological design of the study. JLS interpreted the data and results and drafted the manuscript. HT, MG, AS, ANT and MY were responsible for the critical review of the different versions, making major contributions All authors read and approved the final manuscript.

\section{Acknowledgements}

The authors are grateful for the participation and time of the mothers and infants in the study and the time and efforts of the personnel of the participating clinics.

\section{References}

[1] M. G. Fowler et al., "Benefits and Risks of Antiretroviral Therapy for Perinatal HIV Prevention.," N. Engl. J. Med., vol. 375, no. 18, pp. 1726-1737, 2016.

[2] F. Dabis and E. R. Ekpini, "HIV-1/AIDS and maternal and child health in Africa.," Lancet (London, England), vol. 359, no. 9323, pp. 2097-104, Jun. 2002.

[3] UNAIDS, "Global HIV \& AIDS statistics - 2019 fact sheet," 2019. [Online]. Available: https://www.unaids.org/en/resources/fact-sheet. [Accessed: 23-Oct-2019]. 
[4] S. M. Stricker et al., "Retention in care and adherence to ART are critical elements of HIV care interventions.," AIDS Behav., vol. 18 Suppl 5, no. S5, pp. S465-75, Oct. 2014.

[5] P. M. Barker, W. Mphatswe, and N. Rollins, "Antiretroviral Drugs in the Cupboard Are Not Enough: The Impact of Health Systems' Performance on Mother-to-Child Transmission of HIV.," J.Acquir.Immune.Defic.Syndr., vol. 00, no. 0, pp. 1-4, 2010.

[6] E. L. Sibanda, I. V.D. Weller, J. G. Hakim, and F. M. Cowan, "The magnitude of loss to follow-up of HIV-exposed infants along the prevention of mother-to-child HIV transmission continuum of care," Aids, vol. 27, no. 17 , pp. 2787-2797, 2013.

[7] M. P. Fox et al., "2015 Progress Report on the Global Plan," PLoS One, vol. 11, no. 1, pp. 18-20, 2016.

[8] C. Psaros, J. E. Remmert, D. R. Bangsberg, S. A. Safren, and J. A. Smit, "Adherence to HIV care after pregnancy among women in sub-Saharan Africa: falling off the cliff of the treatment cascade.," Curr. HIV/AIDS Rep., vol. 12, no. 1, pp. 1-5, Mar. 2015.

[9] B. A. Knettel et al., "Retention in HIV Care During Pregnancy and the Postpartum Period in the Option B+ Era," JAIDS J. Acquir. Immune Defic. Syndr., vol. 77, no. 5, pp. 427-438, Apr. 2018.

[10] S. Gumede-Moyo, S. Filteau, T. Munthali, J. Todd, and P. Musonda, "Implementation effectiveness of revised (post-2010) World Health Organization guidelines on prevention of mother-to-child transmission of HIV using routinely collected data in sub-Saharan Africa: A systematic literature review," Medicine (Baltimore)., vol. 96, no. 40, pp. 1-12, 2017.

[11] W. M. Bezabhe, L. Chalmers, L. R. Bereznicki, and G. M. Peterson, "Adherence to Antiretroviral Therapy and Virologic Failure: A Meta-Analysis.," Medicine (Baltimore)., vol. 95, no. 15, p. e3361, Apr. 2016.

[12] C. L. Wallis, C. Godfrey, J. E. Fitzgibbon, and J. W. Mellors, "Key Factors Influencing the Emergence of Human Immunodeficiency Virus Drug Resistance in Low- and Middle-Income Countries," J. Infect. Dis., vol. 216, no. suppl_9, pp. S851-S856, Dec. 2017.

[13] S. Bertagnolio et al., "Determinants of HIV drug resistance and public health implications in lowand middle-income countries," Antivir. Ther., vol. 17, no. 6, pp. 941-953, 2012.

[14] E. Lathrop, D. J. Jamieson, and I. Danel, "HIV and maternal mortality.," Int. J. Gynaecol. Obstet., vol. 127, no. 2, pp. 213-5, Nov. 2014.

[15] S. Linnemayr and T. Rice, "Insights From Behavioral Economics to Design More Effective Incentives for Improving Chronic Health Behaviors, With an Application to Adherence to Antiretrovirals," JAIDS J. Acquir. Immune Defic. Syndr., vol. 72, no. 2, pp. e50-e52, Jun. 2016. 
[16] N. K. Taylor and A. M. Buttenheim, "Improving utilization of and retention in PMTCT services: Can behavioral economics help?," BMC Health Serv. Res., vol. 13, no. 1, p. 406, Dec. 2013.

[17] I. V Bassett et al., "Financial incentives to improve progression through the HIV treatment cascade," Curr Opin HIV AIDS., vol. 10, no. 6, pp. 451-463, 2016.

[18] D. Operario, C. C. Kuo, S. G. Sosa-Rubí, and O. Gálarraga, "Conditional Economic Incentives for Reducing HIV Risk Behaviors: Integration of Psychology and Behavioral Economics," Heal. Psychol., vol. 32, no. 9, pp. 932-940, 2013.

[19] A. Fiszbein and N. R. Schady, “Conditional cash transfers: A World Bank Policy Report," Washington, D.C., 2009.

[20] S. Frederick, G. Loewenstein, and T. O 'donoghue^, "Time Discounting and Time Preference: A Critical Review," foumal ofEconimiic Lit., 2002.

[21] M. Swann, "AIDS Care Psychological and Socio-medical Aspects of AIDS/HIV Economic strengthening for retention in HIV care and adherence to antiretroviral therapy: a review of the evidence Economic strengthening for retention in HIV care and adherence to antiretrovir," AIDS Care - Psychol. Socio-Medical Asp. AIDS/HIV, vol. 30, no. 3, pp. 99-125, 2018.

[22] S. I. McCoy et al., "Cash vs. food assistance to improve adherence to antiretroviral therapy among HIV-infected adults in Tanzania," Aids, vol. 31, no. 6, pp. 815-825, 2017.

[23] M. Yotebieng et al., "Conditional Cash Transfers to Increase Retention in PMTCT Care, Antiretroviral Adherence, and Postpartum Virological Suppression," JAIDS J. Acquir. Immune Defic. Syndr., 2016.

[24] S. S. Solomon et al., "Voucher Incentives Improve Linkage to and Retention in Care Among HIVInfected Drug Users in Chennai, India," Clin. Infect. Dis., vol. 59, no. 4, pp. 589-595, Aug. 2014.

[25] N. M. Petry, C. J. Rash, S. Byrne, S. Ashraf, and W. B. White, "Financial reinforcers for improving medication adherence: findings from a meta-analysis.," Am. J. Med., vol. 125, no. 9, pp. 888-96, Sep. 2012.

[26] M. I. Rosen et al., "Improved Adherence with Contingency Management," AIDS Patient Care STDS, vol. 21, no. 1, pp. 30-40, Jan. 2007.

[27] J. L. Sorensen et al., "Voucher reinforcement improves medication adherence in HIV-positive methadone patients: a randomized trial.," Drug Alcohol Depend., vol. 88, no. 1, pp. 54-63, Apr. 2007.

[28] M. O. Rigsby et al., "Cue-dose training with monetary reinforcement: pilot study of an antiretroviral adherence intervention.," J. Gen. Intern. Med., vol. 15, no. 12, pp. 841-7, Dec. 2000. 
[29] O. Galárraga, B. L. Genberg, R. A. Martin, M. B. Laws, and I. B. Wilson, “Conditional economic incentives to improve HIV treatment adherence: literature review and theoretical considerations," AIDS Behav., vol. 17, no. 7, pp. 2283-2292, 2013.

[30] M. Yotebieng et al., "Conditional cash transfers increase uptake of and retention in PMTCT care: A randomized controlled trial," Lancet HIV, vol. 3, no. 2, pp. e85-e93, 2016.

[31] K. Kroenke, R. L. Spitzer, and J. B. W. Williams, "The PHQ-9: Validity of a brief depression severity measure," J. Gen. Intern. Med., vol. 16, no. 9, pp. 606-613, 2001.

[32] G. Bickel, M. Nord, C. Price, W. Hamilton, and J. Cook, "Guide to Measuring Household Food Security," USDA Econ. Res. Serv., pp. 1-82, 2000.

[33] L.-W. Chao, H. Szrek, N. S. Pereira, and M. V Pauly, "Time preference and its relationship with age, health, and survival probability.," Judgm. Decis. Mak., vol. 4, no. 1, pp. 1-19, Feb. 2009.

[34] J. Haushofer and E. Fehr, "On the psychology of poverty," Science, pp. 862-867, 2014.

[35] P. D. Sozou and R. M. Seymour, "Augmented discounting: interaction between ageing and timepreference behaviour," Proc. R. Soc. B Biol. Sci., vol. 270, no. 1519, pp. 1047-1053, May 2003.

[36] A. Ahmed, "Rationality and Future Discounting," Topoi, vol. 1, no. 0123456789, pp. 1-12.

[37] F. G. Moeller, D. M. Dougherty, E. S. Barratt, J. M. Schmitz, A. C. Swann, and J. Grabowski, "The impact of impulsivity on cocaine use and retention in treatment.," J. Subst. Abuse Treat., vol. 21, no. 4, pp. 193-8, Dec. 2001.

[38] L. Stevens, A. Verdejo-García, A. E. Goudriaan, H. Roeyers, G. Dom, and W. Vanderplasschen, "Impulsivity as a vulnerability factor for poor addiction treatment outcomes: A review of neurocognitive findings among individuals with substance use disorders," J. Subst. Abuse Treat., vol. 47, no. 1, pp. 5872, Jul. 2014.

[39] S. Linnemayr and C. Stecher, "Behavioral Economics Matters for HIV Research: The Impact of Behavioral Biases on Adherence to Antiretrovirals (ARVs).," AIDS Behav., vol. 19, no. 11, pp. 2069-75, Nov. 2015.

[40] H. Thirumurthy et al., "Time Preferences Predict Mortality among HIV-Infected Adults Receiving Antiretroviral Therapy in Kenya.," PLoS One, vol. 10, no. 12, p. e0145245, 2015.

[41] E. Hamilton et al., "Using the PMTCT Cascade to Accelerate Achievement of the Global Plan Goals," JAIDS J. Acquir. Immune Defic. Syndr., vol. 75, pp. S27-S35, 2017.

[42] P. M. Musumari, M. D. Feldman, T. Techasrivichien, E. Wouters, M. Ono-Kihara, and M. Kihara, "'If I have nothing to eat, I get angry and push the pills bottle away from me': A qualitative study of patient 
determinants of adherence to antiretroviral therapy in the Democratic Republic of Congo," AIDS Care, vol. 25, no. 10, pp. 1271-1277, Oct. 2013.

[43] B. Mupenda et al., "Terms Used for People Living With HIV in the Democratic Republic of the Congo," Qual. Health Res., vol. 24, no. 2, pp. 209-216, Feb. 2014.

[44] M. Yotebieng et al., "Conditional cash transfers improve retention in PMTCT services by mitigating the negative effect of not having money to come to the clinic," JAIDS J. Acquir. Immune Defic. Syndr., vol. 74, no. 2, p. 1, 2016.

[45] S. Burks, J. Carpenter, L. Götte, and A. Rustichini, "Which measures of time preference best predict outcomes: Evidence from a large-scale field experiment," J. Econ. Behav. ..., no. 5808, 2012.

[46] D. Laibson, "Golden Eggs and Hyperbolic Discounting," Q. J. Econ., vol. 112, no. 2, pp. 443-477, 1997.

[47] T. O’Donoghue and M. Rabin, "Doing It Now or Later," Am. Econ. Rev., vol. 89, no. 1, pp. 103-124, Mar. 1999.

[48] K. J. Rothman, S. Greenland, and T. L. Lash, Modern epidemiology. Philadelphia: Wolters Kluwer Health/Lippincott Williams \& Wilkins, 2008.

[49] K. Esopo et al., "Measuring self-efficacy, executive function, and temporal discounting in Kenya," Behav. Res. Ther., vol. 101, pp. 30-45, Feb. 2018.

[50] L. Carvalho, S. Meier, and S. Wang, "Poverty and Economic Decision-Making: Evidence from Changes in Financial Resources at Payday.," Am. Econ. Rev., 2014.

[51] R. Cassidy, G. Rasool, T. Khan, and H. Zaman, "Are the poor really so present-biased? Experimental evidence from Pakistan," Inst. Fisc. Stud., vol. 18, no. 24, 2017.

[52] F. C. Mukumbang, S. Van Belle, B. Marchal, and B. van Wyk, "Exploring 'generative mechanisms' of the antiretroviral adherence club intervention using the realist approach: a scoping review of researchbased antiretroviral treatment adherence theories," BMC Public Health, vol. 17, no. 1, p. 385, Dec. 2017.

[53] National Institutes of Health (NIH), "Science of Behavior Change," 2018. [Online]. Available: https://commonfund.nih.gov/behaviorchange. [Accessed: 10-Nov-2018].

\section{Supplementary Files}

This is a list of supplementary files associated with this preprint. Click to download.

- STROBEchecklistv4cohort.pdf 